\title{
Air-to-Air Collaborative Learning: A Multi-Task Orchestration in Federated Aerial Computing
}

\author{
Uchechukwu Awada*, Jiankang Zhang*†, Sheng Chen ${ }^{\ddagger}$ and Shuangzhi Li* \\ *School of Information Engineering, Zhengzhou University, Zhengzhou 450001, China \\ ${ }^{\dagger}$ Department of Computing and Informatics, Bournemouth University, Poole BH12 5BB, UK \\ ${ }^{\ddagger}$ School of Electronics and Computer Science, University of Southampton, Southampton SO17 1BJ, UK \\ Email: awada@gs.zzu.edu; jzhang3@bournemouth.ac.uk; sqc@ecs.soton.ac.uk; ielsz@zzu.edu.cn
}

\begin{abstract}
Recent research on edge computing (EC) has proposed federated or collaborative learning technique, where machine learning models are shared among participating edge deployments, thereby benefiting from all available datasets without exchanging them. In addition, EC systems are currently exploiting attaching portable edge devices on drones for data processing close to the sources, to achieve high performance, fast response times and real-time insights. Existing research lack the potential to federate edge resources and manage corresponding service entities running across multiple drones, thus resulting to suboptimal performance. Therefore, we introduce AerialEdge, a federated learning-based orchestration framework for a federated aerial EC system. We propose a federated multi-output linear regression model to estimate multi-task resource requirements and execution time, to select the closest drone deployment having congruent resource availability and flight time to execute ready tasks at any given time. For better utilization of resources, we propose a variant bin-packing optimization approach through gang-scheduling of multi-dependent containerized tasks that coschedules and co-locates tasks tightly on nodes to fully utilize available resources. Extensive experiments on real-world datatrace from Alibaba cluster trace with information on task dependencies show the effectiveness, fast executions, and resource efficiency of our approach.
\end{abstract}

Index Terms-Edge computing, dependency-aware, federated learning, edge federation, execution time, resource efficiency

\section{INTRODUCTION}

Emerging technologies, such as connected and autonomous vehicles (CAVs), healthcare IoT systems, real-time augmented reality, smart cities, Industry 4.0, etc, rely on edge computing resources, to offload their computational intensive tasks, improve response times and achieve real-time insights. To this end, Cloud Computing providers, such as Amazon Web Services (AWS), Microsoft Azure, Alibaba Cloud, Google Cloud, Dell Technologies Cloud, IBM Cloud, etc., have recently introduced various portable edge devices and begun offering cloud computing services directly on these edge devices. Examples of these portable edge devices include, AWS Snowcone [1] (with 8TB, 2vCPU and 4GiB of resources), HIVECELL [2] (with 500GB 6vCPU 8GiB of resources), etc. Each weighs about $2.1 \sim 3 \mathrm{~kg}$ and capable of accelerated artificial intelligent (AI) inferencing.

Consequently, edge computing (EC) systems are recently exploiting attaching these portable edge devices on low altitude platform (LAP) unmanned aerial vehicles (UAVs) or drones as aerial deployments, to execute complex resourcehungry use cases. A state-of-the-art drone technology, called Drone-in-a-box [3], is most suitable for aerial EC deployment. A drone-in-a-box system can be deployed autonomously from a box that serves as a landing pad and charging base. After executing all its tasks, it returns to its box. However, a typical drone has a limited flight time due to power factor which can lead to loss of job if it is not taking into consideration [4]. The critical issue is how to optimize both the drones' flight time and application execution on the attached edge device(s) in a timely manner, without jeopardizing application performance.

Most recently, research on EC has proposed a machine learning (ML) technique that trains an algorithm across multiple edge deployments holding local data samples, without exchanging them [5], [6]. This is called Federated or Collaborative Learning. Models learned in such a way promise to greatly improving usability by powering more functionalities and intelligent applications [7]. However, efficient orchestration of complex dependencies among tasks in such individual deployments is challenging due to constrained resource capabilities, vendor lock-in, availability factors, etc. Existing researches on edge federated/collaborative learning, i.e., [5], [6] do not consider the ability to keep edge resources running across different edge deployments in a single pool, such that these resources can be holistically managed and controlled from a single federated plane, vendor lock-in situations can be eliminated, and applications can be deployed dynamically across the resources. Note that edge FL technique produces and shares a single global inference model among participating edge through a remote/central server, while the merging of resources among participating edge, such that these resources are holistically managed and controlled from a single federated plane is referred to as Edge Federation (EF). EF minimizes latency by serving users from the edge deployment that is the closest to them [8].

Nevertheless, to execute complex applications (i.e., multidependent tasks, where each task has diverse resource request) pose several challenges: (i) Given a federated clusters running across multiple drones, to automatically decide where a job or multi-task should be deployed is a challenging task. Previous works [9], [10] assume that each server can only execute one task or job at any time and schedules each task individually, which could result to higher communication overhead. (ii) The 
default schedulers deploy tasks randomly on nodes (virtual machines) that have sufficient resource availability without considering dependent tasks, which results in longer execution time, resource wastage through underutilized nodes, and a reduction in the number of tasks that can be executed, given the available resources. In addition, it does not pack tasks tightly on available resources in order to achieve high utilization.

This motivates our research to address these limitations and to extend the state-of-the-arts by proposing AerialEdge, which considers jointly task dependencies, heterogeneous resource demands, and flight times for drones in a federated autonomous aerial EC-enabled learning system. Specifically, we propose a federated multi-output linear regression model to estimate multi-task resource requirements and execution time, and a multi-task dispatching policy called Closest to select the closest drone deployment having congruent resource availability and flight time to execute ready tasks at a given time and to autonomously deploy the selected drone to the needed location. We also propose a variant bin-packing optimization through gang-scheduling of multi-dependent tasks that co-schedules and co-locates multi-tasks tightly on nodes to fully utilize available resources. We show that the proposed AerialEdge can minimize the actual completion time of tasks using minimum resources, such that the actual completion time is much less than the drones' flight time. In summary, to achieve our AerialEdge implementation, we address the following critical areas:

- Federated aerial edge state service: to stream all changes in the drones and the attached edge devices across the federated deployments, and to enable us to query the state of drones, in terms of flight time availability, and resource availability, so that informed decisions can be made on multi-task orchestration and co-location.

- Dynamic optimization strategy: to efficiently manage tasks across the federated edge resources or clusters, and to enable us to co-locate multi-tasks for fully utilizing available resources [11], [12].

- Extensive experimentation and comparison: to evaluate AerialEdge on the real-world Alibaba Cluster data trace, and to compare it with existing deployment approaches.

\section{Preliminaries AND OUR Motivation}

In the light of the prior research, we first present some preliminaries and related work, and discuss our motivation.

\section{A. Edge Federated Learning}

The main goal of edge FL is to collaboratively learn a model from the data $\mathcal{D}_{1}, \cdots, \mathcal{D}_{N}$, stored across $N$ distributed deployments, where each dataset $\mathcal{D}_{i}=\left\{\left(\mathrm{x}_{i, j}, \mathrm{y}_{i, j}\right)\right\}_{j=1}^{n_{i}}$ contain $d$-dimensional tensors of data features $\mathrm{x}_{i, j} \in \mathbb{R}^{1 \times d}$ and $c$ dimensional tensor data labels $\mathrm{y}_{i, j} \in \mathbb{R}^{1 \times c}$. A conventional method is to aggregate the datasets in one server or datacenter, i.e., $\mathcal{D}=\mathcal{D}_{1} \cup \mathcal{D}_{2} \cup \cdots \cup \mathcal{D}_{N}$, and utilize it to train a model $\theta_{\mathrm{S}}$. In edge FL, the participating members/deployments independently train their models $\theta_{L_{i}}$ based on their local datasets $\mathcal{D}_{i}$. Then at time $t \geq 0$, the participating deployments

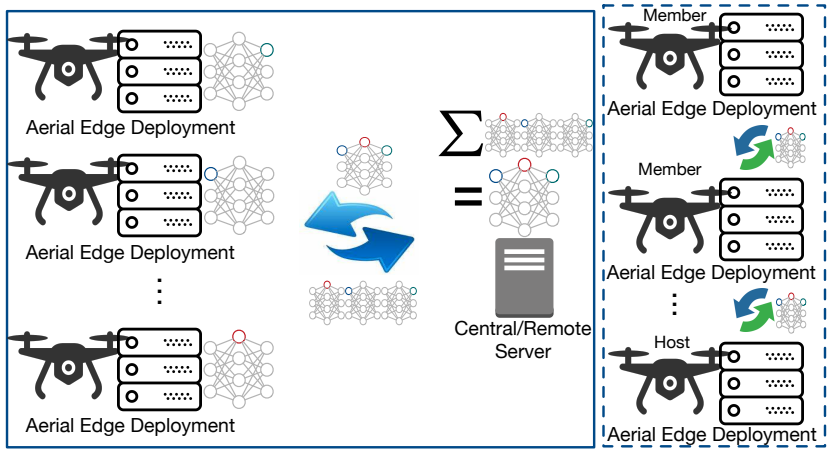

(a) FL system

(b) EF system

Fig. 1: (a) The architecture of edge federated learning system, (b) The architecture of a merged edge-enabled federated learning system.

send their models $\theta_{L_{i}}, 1 \leq i \leq N$, to the remote/central server, where the global update $\theta_{G}$ is computed by aggregating all the deployments models:

$$
\theta_{G}^{t}=\sum_{i=1}^{N} \theta_{L_{i}}
$$

In return, the global model is distributed to the participants, where it is used to update the current local models. Let the updated local models be $\theta_{L_{1}}^{t}, \theta_{L_{2}}^{t}, \ldots, \theta_{L_{N}}^{t}$, and therefore the update at the $i$ th deployment can be expressed as $\Theta_{L_{i}}^{t}=\theta_{L_{i}}^{t}-$ $\theta_{L_{i}}$. At time $t+1$, the updates $\Theta_{L_{i}}^{t}, 1 \leq i \leq N$, from the participants are sent back to the central server, where a global update is computed:

$$
\theta_{G}^{t+1}=\theta_{G}^{t}+\sum_{i=1}^{N} \Theta_{L_{i}}
$$

For deployment $i$ with dataset $\mathcal{D}_{i}$, the learning problem is to solve the following optimization:

$$
\theta_{L_{i}}^{\star}=\arg \min _{\theta_{L_{i}} \in \mathbb{R}^{d \times c}} \frac{1}{2 n} \sum_{j=1}^{n_{i}}\left\|\mathrm{x}_{i, j} \theta_{L_{i}}-\mathrm{y}_{i, j}\right\|_{2}^{2}+\frac{\lambda}{2}\left\|\theta_{L_{i}}\right\|_{F}^{2},
$$

where $\lambda$ is the regularization parameter and $\|\cdot\|_{F}$ denotes the Frobenius norm. The optimization (3) is commonly solved using gradient-descent techniques, where the model is updated sequentially until convergence, i.e., $\theta_{L_{i}}^{l+1}=\theta_{L_{i}}^{l}-$ $\eta\left(\frac{1}{n} \mathbf{g}\left(\theta_{L_{i}}^{l}\right)+\lambda \theta_{L_{i}}^{l}\right)$, in which $\eta$ is the learning rate, $\mathbf{g}\left(\theta_{L_{i}}\right)=$ $\frac{1}{n} \mathbf{X}_{i}^{\mathrm{T}}\left(\mathbf{X}_{i} \theta_{L_{i}}-\mathbf{Y}_{i}\right)$ denotes the gradient of the loss function, and $\mathbf{X}_{i}=\left[\mathrm{x}_{i, 1}^{\mathrm{T}} \cdots \mathrm{x}_{i, n_{i}}^{\mathrm{T}}\right]^{\mathrm{T}}$ and $\mathbf{Y}_{i}=\left[\mathrm{y}_{i, 1}^{\mathrm{T}} \cdots \mathrm{y}_{i, n_{i}}^{\mathrm{T}}\right]^{\mathrm{T}}$ are the local feature set and label set, respectively.

Although FL promises to produce a high-quality global model, however there is no resource coordination among the participants.

\section{B. Merged Edge-Enable Federated Learning}

This research focuses on Job orchestration in merged/federated edge computing enabled learning system, as shown in Fig 1 (b). In the merged edge-enabled FL approach, participating deployments can potentially merge 


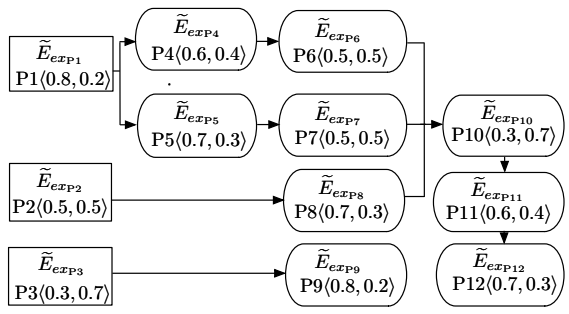

Fig. 2: DAG of a video processing job.

their resources, such that all the resources running across different edge deployments can be holistically managed and controlled, and applications can be deployed dynamically across the resources. In a merged edge clusters setup, the Federation Control Plane (FCP) is deployed on one of the clusters which serves as the host cluster. Participating clusters or members can be added or removed from the FCP. Owing to the merged resources, we will assume that the datasets $\mathcal{D}_{i}$ across distributed deployments share the same feature and label space, i.e.,

$$
\mathrm{x}_{i_{1}, j}=\mathrm{x}_{i_{2}, j}, \mathrm{y}_{i_{1}, j}=\mathrm{y}_{i_{2}, j}, \forall \mathcal{D}_{i_{1}}, \mathcal{D}_{i_{2}}, i_{1} \neq i_{2} .
$$

In this paper, we propose a merged edge-enabled FL system, whereby the participating deployments can collaboratively learn a shared global model through the host cluster, thereby eliminating the need for a central server. That is, the host cluster serves as the models aggregator. We propose the use of this shared model, a multi-output linear regression model, to estimate multi-task resource requirement and execution time, for the selection of the closest deployment within the federation having congruent resource availability and flight time to execute ready tasks at any given time.

\section{Task Dependency-Awareness}

Dependency-awareness is critical for achieving efficient multi-task orchestration, i.e., dispatching and co-location. Most of the batch workloads of Alibaba cluster trace [13] for example are directed acyclic graphs (DAGs), and only some of them are independent. The average Alibaba cluster trace job has a dependency depth of 10. A job typically consists of several tasks whose dependencies are expressed by DAG. Clearly, if a task $A$ is depending on task $B$, then task $A$ cannot start until all the instances of task $B$ are completed. For example, the DAG of a video processing job is shown in Fig. 2. The Job consists of 12 tasks. Knowledge about task characteristics, such as resource demands and dependencies, is necessary to pack or co-locate tasks effectively in a node or cluster, ultimately to minimize the response times and improve resource utilization [11], [14]. Hence a key objective is to reduce the execution time of such tasks and to improve resource utilization by considering the inter-task dependency and resource demands.

\section{Our Motivation}

To illustrate the advantage of AerialEdge, we show a motivating example using Fig. 2. It shows each task of a video processing job, with its estimated execution time $\widetilde{E}_{e x_{i}}$ and estimated resource demand (CPU and memory $\langle c, m\rangle$ ). Our aim is to dispatch this job to the closest deployment with requisite available resources and flight time, such that dependent tasks can communicate faster to make the applications more interactive, compared to other deployments across different clusters. Here, we assume that the deployment has the requisite available resources to accommodate all the tasks, i.e., $\langle 7,5\rangle$. We illustrate the scheduling approach AerialEdge and its actual execution time together with three other state-of-the-art approaches, namely, Spear [14], Graphene [15] and Tetris [12], as well as the random approach. Our AerialEdge achieves the lowest actual execution time of $\sum_{i=1}^{n} E_{e x_{i}} / n$ ( $n$ is the number of tasks), due to the following reasons: (i) our approach utilizes gang scheduling [16], which co-schedules all the tasks at a time, and (ii) our packing strategy explores the available nodes to find the best one which has requisite available resources (CPU and memory) to execute all the tasks by packing or co-locating them tightly on the node. By contrast, Spear and Tetris deploy the same tasks individually or in parts, resulting in execution times of $\sum_{y=1}^{n} E_{e x_{y}}+\sum_{z=1}^{m} \sum_{i=1}^{k} E_{e x_{i z}} / k$ and $\sum_{z=1}^{m} \sum_{i=1}^{n} E_{e x_{i z}} / n$, respectively. In particular, Spear picks tasks along the critical path (CP) in the DAG. The CP of a task is the longest path from the task to the output. As an example, given a job with 100 DAGs, Spear deploys about $15 \%$ of the tasks at a time. Tetris on the other hand does not consider the task dependencies. It deploys at least $50 \%$ of any given tasks at a time and focuses on packing tasks on nodes to achieve high resource utilization. Graphene, a state-of-the-art dependency-aware scheduler, considers both task dependencies and resource packing. It first co-schedules some tasks identified as troublesome tasks and then places the rest of the tasks afterwards, resulting in an execution time of $\sum_{x=1}^{n} E_{e x_{x}}+\sum_{z=1}^{m} \sum_{i=1}^{k} E_{e x_{i z}} / k$. The random approach deploys a task randomly to any available node, and assumes a node can only execute a task at a time, resulting in an execution time of $\sum_{i=1}^{n} E_{e x_{i}}$. Generally, delay in scheduling dependent tasks directly impacts job completion time, and utilizing gang scheduling is beneficial for overall performance.

\section{Proposed AerialEdge}

In this section, we detail our proposed AerialEdge for achieving high resource utilization and minimizing the execution times of applications deployed in federated aerial edge resources. Our system model is depicted in Fig. 3 .

\section{A. System Model}

Federated learning takes advantage of historical data across participating deployments to produce a high-level model. As multi-dependent containerized applications are admitted into the system, their resource requirements and execution time are estimated using linear regression model, in this case a multioutput linear regression model. The model is run on the multitask features $\mathbb{F}$, such as type of tasks $\epsilon$, dependency depth $\gamma$, data size $\delta$, to estimate the values, i.e.,

$$
\Theta(\mathbb{F})=\left\{\left(\widetilde{T}_{i}^{\langle c, m\rangle}, \widetilde{E}_{e x_{i}}\right)\right\}_{i=1}^{\gamma},
$$




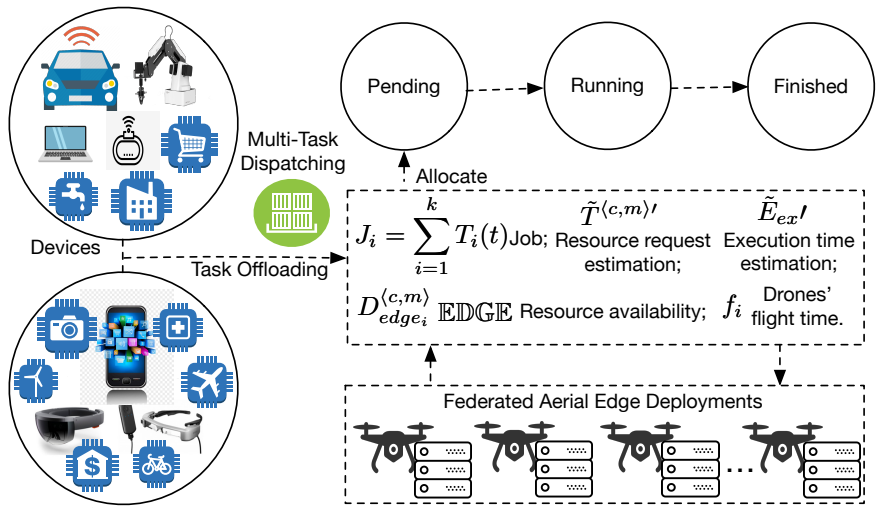

Fig. 3: Orchestration overview of AerialEdge.

where $\widetilde{T}^{\langle c, m\rangle}$ is the estimated resource requirements (in terms of CPU and memory) and $\widetilde{E}_{e x}$ is the estimated execution time, and then to deploy it to the closest edge with congruent resource availability and flight time. To build this predictor $\Theta$, we train a ML model based on Keras [17] with historical data from previously executed tasks/jobs. Keras is a library which wraps TensorFlow [18] complexity into simple and user-friendly API. The advantage of using containers to host applications at the edge is that these applications can be executed within its estimated resource requirement $\langle\delta, c, m\rangle$, where $\delta$ denotes the size of data, $c$ denotes CPU requirement and $m$ denotes memory requirement, in any edge deployment regardless of the resource type, configuration or vendor/provider.

Therefore, given a federated aerial edge deployment $\mathbb{E} \mathbb{D} \mathbb{G} \mathbb{E}$, where each individual edge deployment $D_{\text {edge }_{i}}$ is a cluster of container-instances (i.e., edge device(s) with virtualized container-optimized nodes) with total resource capacity or availability $D_{\text {edge }_{i}}^{\langle\delta, c, m\rangle}$, our aim is to deploy ready applications, $\mathbb{C}$, to the closest drone deployment with minimum flight time $f_{i}^{l}$ from its box to the location $l$, having sufficient flight time $f_{i}$ and resource capacity or availability to execute the tasks, such that the tasks are executed concurrently, namely,

$$
\mathbb{C} \Rightarrow D_{\text {edge }_{i^{\star}}},
$$

where

$D_{\text {edge }_{i^{\star}}}=\arg \min _{D_{\text {edge }} \in \mathbb{E} \mathbb{D G E}}\left\{f_{i}^{l}: f_{i}^{l}<f_{i}, D_{\text {edge }_{i}}^{\langle\delta, c, m\rangle}\right.$ sufficient $\}$.

Existing works on UAV or drone-based task offloading and execution in EC systems [19], [20] do not consider drones' flight time and assume a drone can fly for unlimited amount of time, which can lead to loss of job due to drones' limited flight time [4]. Therefore, given a federated edge deployment $\mathbb{E D G} \mathbb{E}$ and a set $\mathbb{C}$ of inter-dependent containerized applications, where each application $T \in \mathbb{C}$ serves as a task with its estimated resource demand and execution time denoted as $\widetilde{T}^{\langle c, m\rangle}$ and $\widetilde{E}_{e x}$ respectively, our goal is to use the estimated values for all the tasks to select an edge having congruent resource availability and flight time, such that we can intelligently schedule the tasks to minimize the actual execution time as well as to achieve high resource utilization efficiency.
For a task $T$, let $E_{s}$ and $E_{c}$ denote its actual starting time and completion time, respectively. Therefore, the actual execution time of $T$ is:

$$
E_{e x}=E_{c}-E_{s}
$$

AerialEdge utilizes the gang scheduling [16] strategy to coschedule all ready applications at a time. Hence the aggregate execution time of a multi $(n)$-task $\mathbb{C}$ is given as $\sum_{i=1}^{n} \frac{E_{e x_{i}}}{n}$. The federated edge system $\mathbb{E D G E}$ consists of all $N$ participating individual edge deployments $D_{\text {edge }_{i}}, 1 \leq i \leq N$, i.e.,

$$
\mathbb{E D G E}=\sum_{i=1}^{N} D_{\text {edge }_{i}} .
$$

Given a cluster of container-instances or nodes $I$ in each deployment $D_{\text {edge }_{i}}$, let $I_{i}^{\langle c, m\rangle}$ denote each node's resource capacity or availability. For the purpose of simplicity, we will focus on the CPU and memory requirements/capacity of all tasks and resources. That is, the storage is sufficient for the size of data input $\delta$, and hence the requirement $\langle\delta, c, m\rangle$ is simplified as $\langle c, m\rangle$. The estimated resource demands and execution time of $k$ containerized applications to be orchestrated, $\sum_{i=1}^{k} \widetilde{T}_{i}^{\langle c, m\rangle}$ and $\sum_{i=1}^{k} \widetilde{E}_{e x_{i}}$, the update state of the $\mathbb{E} \mathbb{D} \mathbb{G} \mathbb{E}$ clusters, i.e., the resources availability $D_{\text {edge }}^{\langle c, m\rangle}$, and drones' flight time $f_{i}$ are important information needed in order to make informed decision on where to deploy ready applications $\mathbb{C}$ at time $t$. Our strategy chooses the closest edge having requisite capacity and fight time. In real scenario where multi-users $u \in \mathbb{U}$ offload multi-tasks with multi-dependency at $t$, these applications are deployed as a multi-Job $\mathbb{J}$, where each Job $J$ is a collection of each user's multi-tasks, with collective resource demand estimation denoted as $\sum_{i=1}^{k} \widetilde{T}_{i}^{\langle c, m\rangle}=\widetilde{T}^{\langle c, m\rangle \prime}$, and the aggregate execution time estimation as $\sum_{i=1}^{k} \widetilde{E}_{e x_{i}}=\widetilde{E}_{e x}$. We

TABLE I: Common Notations

\begin{tabular}{|l|l|}
\hline \hline Notation & Description \\
\hline $\mathbb{E D} \mathbb{G} \mathbb{E}$ & Federated edge deployments \\
\hline$T$ & Individual application or task \\
\hline$\langle\delta, c, m\rangle$ & Storage, CPU and memory resources \\
\hline $\mathbb{C}$ & A set of containerized applications \\
\hline$\widetilde{T}^{\langle c, m\rangle}$ & Task resource requirements estimation \\
\hline$D_{e d g e_{i}}$ & Individual edge deployment or cluster \\
\hline$D_{e d g e_{i} \star}$ & Closest edge deployment or cluster \\
\hline$I_{i}$ & Container-instance or node in a cluster \\
\hline$I_{i}^{\langle c, m\rangle}$ & Resource capacity or availability of a node \\
\hline$D_{e d g e_{i}}^{\langle c, m\rangle}$ & Resource capacity/availability in an edge \\
\hline$D_{e d g e_{i} U}^{\langle c, m\rangle}$ & Resources used for execution \\
\hline$D_{e d g e_{i}}^{\langle c, m\rangle}$ & Actual resources usage of jobs \\
\hline$E_{s}, E_{c}$ & Application/task start, completion time \\
\hline$E_{e x}$ & Application or task execution time \\
\hline$\widetilde{E}_{e x}$ & Application or task execution time estimation \\
\hline$f_{i}$ & Drones' flight time \\
\hline$f_{i}^{l}$ & Drones' flight time from its box to location \\
\hline$\rho_{C}^{\langle c, m\rangle}$ & Cluster resource utilization \\
\hline$\rho_{C}^{\langle c\rangle}, \rho_{C}^{\langle m\rangle}$ & Cluster CPU, memory resource utilization \\
\hline$\epsilon{ }_{J}$ & The type of job \\
\hline$\gamma_{J}$ & Dependency depth of a job \\
\hline $\mathbb{F}^{\langle}$ & Aet of multi-task features \\
\hline$J, \mathbb{J}$ & \\
\hline$u, \mathbb{U}$ & A User, A set of Users \\
\hline & \\
\hline
\end{tabular}


can deploy all users' Jobs with dependency on the same cluster by jointly considering $\sum_{J \in \mathbb{J}} \widetilde{T}^{\langle c, m\rangle \prime}, D_{e d g e_{i}}^{\langle c, m\rangle}, \sum_{J \in \mathbb{J}} \widetilde{E}_{e x} \prime$ and $f_{i}$. Therefore, the aggregate of the actual execution time of a multi-job $\mathbb{J}$ is given as:

$$
\sum_{J \in J} \sum_{i=1}^{k} \frac{E_{e x_{i}}}{k}=E_{e x}
$$

and we can deploy a multi-job to the closest edge, such that:

$$
\mathbb{J} \Rightarrow D_{\text {edge }_{i}}{ }
$$

The resource utilization of the cluster for multi-job deployment is thus

$$
\rho_{C}^{\langle c, m\rangle}=\frac{\sum_{J \in \mathbb{J}} \widetilde{T}^{\langle c, m\rangle \prime}}{D_{\text {edge }_{i}}^{\langle c, m\rangle}} .
$$

\section{B. Problem Formulation}

The basic notations adopted are described in Table II AerialEdge includes an intelligent scheduling, which packs tasks tightly on nodes to fully utilize available resources at edge clusters, while considering task dependencies.

Our objectives are to maximize the cluster resource utilization, $\rho_{C}^{\langle c, m\rangle}$ of 12 , and to minimize the overall actual execution time of tasks, $E_{e x}$ of $(10)$, subject to certain constraints.

Constraints: First, the collective resource demand or request estimation of a multi-job $\mathbb{J}$ or multi-task at any given time $t$ cannot exceed the collective resource capacity or available in the cluster:

$$
\sum_{J \in \mathbb{J}} \widetilde{T}^{\langle c, m\rangle \prime} \leq D_{e^{\prime d g e} e_{i}}^{\langle c, m\rangle}, \quad \forall\langle c, m\rangle
$$

Second, the aggregate execution time estimation of a multijob $\mathbb{J}$ or multi-task at any given time $t$ cannot exceed the flight time availability of any selected drone:

$$
\sum_{J \in \mathbb{J}} \widetilde{E}_{e x} \prime \leq f_{i}, \quad \forall D_{\text {edge }_{i}} \in \mathbb{E} \mathbb{D} \mathbb{G} \mathbb{E}
$$

Third, unused or inactive container-instance or node $I_{i} \in$ $D_{\text {edge }_{i}}$ in the cluster would be shut down. All the nodes are in one of the two states: Active and Inactive. An Active node is a node that is ready to accept jobs or has at least a job being started, executing or completing. An Inactive node is a node that is not ready to accept jobs and not having at least a job that is being started, executing or completing. These two states can be expressed as follows:

$$
\forall_{c, m} \beta\left(I_{i}\right)= \begin{cases}1, & \text { Active if } J_{i} \in\left[E_{s}, E_{c}, E_{e x}\right], \\ 0, & \text { Inactive if } J_{i} \notin\left[E_{s}, E_{c}, E_{e x}\right],\end{cases}
$$

where the indicator $\beta\left(I_{i}\right)=1$ indicates that the node $I_{i}$ is ready to accept new jobs, and at least a job $J_{i}$ is being started, executing or completing, i.e., $J_{i} \in\left[E s, E_{c}, E_{e x}\right]$, on $I_{i}$; otherwise $\beta\left(I_{i}\right)=0$.
Optimization formulation: Hence, maximizing utilization of a cluster depends on application orchestration:

$$
\begin{array}{ll}
\text { Maximize } & \rho_{C}^{\langle c, m\rangle}=\frac{\sum_{J \in \mathbb{J}} \widetilde{T}^{\langle c, m\rangle \prime}}{D_{\text {edge }_{i}}^{\langle c, m\rangle}}, \\
\text { subject to } \quad & \mathbb{J} \Rightarrow D_{\text {edge }_{i^{\star}}, \quad \exists,} \\
& \sum_{J \in \mathbb{J}} \widetilde{E}_{e x^{\prime}} \leq f_{i}, \quad \forall_{D_{\text {edge }_{i}} \in \mathbb{E D} \mathbb{G E}}, \quad \exists, \\
& \beta\left(I_{i}\right) \in\{0,1\}, \quad \exists, \\
& \sum_{J \in \mathbb{J}} \widetilde{T}^{\langle c, m\rangle \prime} \leq D_{\text {edge }_{i}}^{\langle c, m\rangle}, \quad \forall\langle c, m\rangle .
\end{array}
$$

On the other hand, the overall actual execution time can be minimized depending on orchestration:

$$
\begin{array}{ll}
\text { Minimize } & \sum_{J \in \mathbb{J}} \sum_{i=1}^{k} \frac{E_{e x_{i}}}{k}=E_{e x} \prime \\
\text { subject to } & \mathbb{J} \Rightarrow D_{\text {edge }_{i^{\star}}}, \quad \forall\langle c, m\rangle \cdot
\end{array}
$$

\section{Algorithm}

Our AerialEdge solution consists of three components: the resource requirement and execution time estimations, dispatching, and packing. The values for multi-job required by the dispatcher are first estimated. Our dispatching strategy is based on the orchestration of ready tasks to the closest cluster or drone deployment with the minimum flight time $f_{i}^{l}$ to arrive at location $l$, and having requisite available resources to accommodate the tasks, while our packing strategy involves packing these tasks tightly on nodes or container-instances to fully utilize the available resources. Below we detail the procedures of the execution time estimation, dispatching, and co-location or packing.

Resource requirement and execution time estimation: When the set of tasks $\mathbb{C}$ are ready to be deployed, the first step is to estimate the collective resource requirement $\widetilde{T}^{\langle c, m\rangle \prime}$ and execution time $\widetilde{E}_{e x} \prime$. We have trained a ML regression model with historical data for this prediction task. The input to the prediction model is the set of multi-task features $\mathbb{F}$, such as type of tasks $\epsilon$, dependency depth $\gamma$, and data size $\delta$, and the output is the resource requirement and execution time estimation. Algorithm 1 describes the estimations for multijob. Once the estimation values are extracted, they are used in the dispatching stage.

Dispatching: Our policy is to dispatch a set of tasks to the closest edge $D_{\text {edge }_{i} \text { * }}$ with the congruent resource capacity or availability and flight time availability for any selected drone, i.e., $T^{\langle c, m\rangle \prime} \cong D_{e^{\prime} d g e_{i^{\star}}}^{\langle c, m\rangle}$ and $\widetilde{E}_{e x}{ }_{\text {ex }} \cong f_{i^{\star}}$, respectively. Our strategy utilizes the closest heuristic to minimize the overall response time. This is based on the orchestration of ready tasks to the closest deployment or cluster having requisite available resources to execute the tasks. Closest is a widely adopted heuristic or principle in distributed systems, since mobile devices often need to communicate only with the closest or nearest edge-clouds. Most of the works on edge-clouds, e.g., [11], [21], adopt the closest principle as the task offloading policy. 

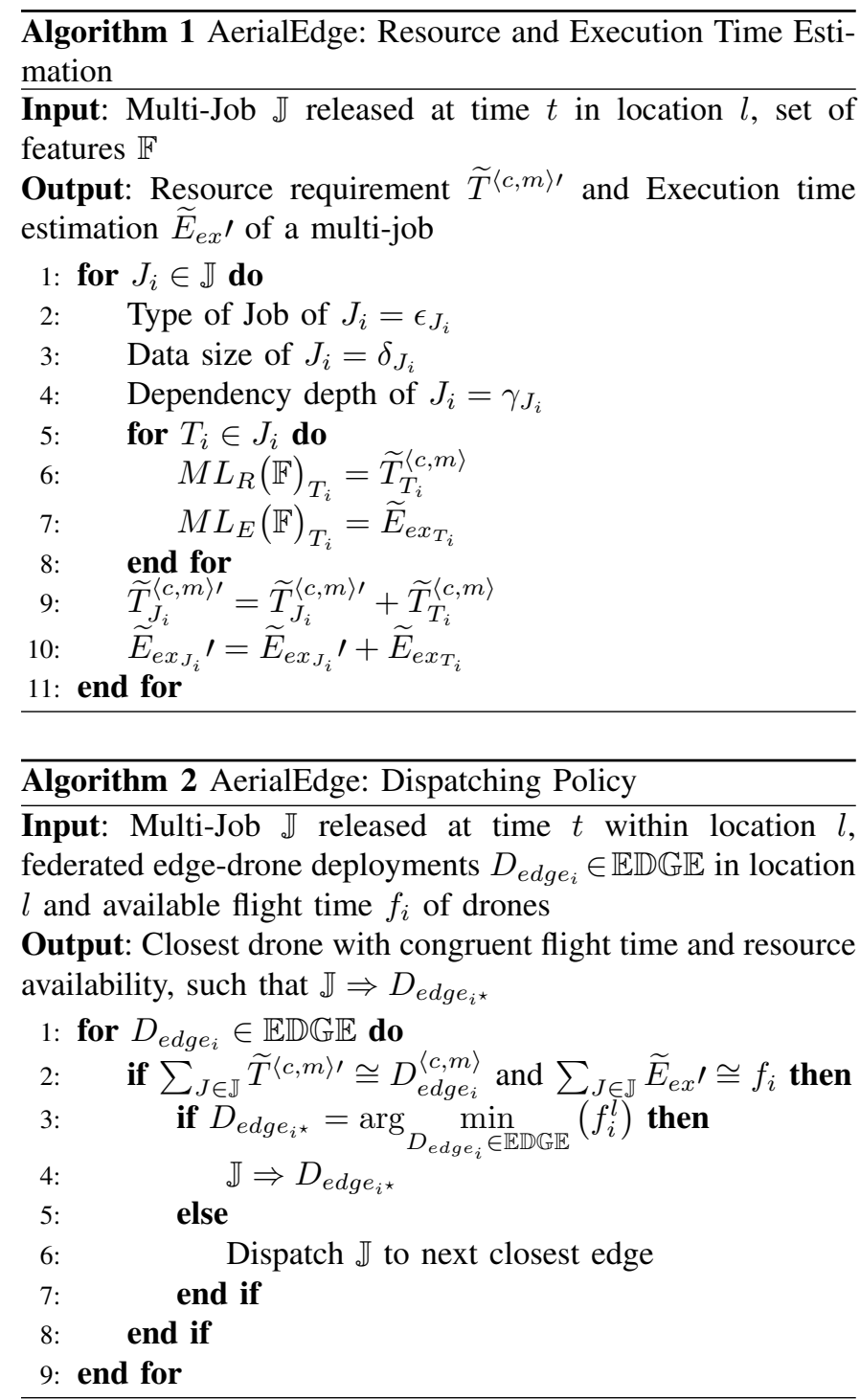

Algorithm 2 describes the dispatching procedure in 3 steps. First, it captures the collective resource demand of ready multitask/job and location of users, and updates the state of $\mathbb{E} \mathbb{D} \mathbb{G E}$ resources. Second, it selects the closest edge having congruent resources (line 3). Lastly, it dispatches the multi-task/job to the selected cluster (line 4). If the closest edge does not have the required resources, the selection procedure is repeated until the next closest edge having congruent resources is found, and the multi-task/job is dispatched to the next closest edge (line 6).

Packing: At the edge cluster, we develop a new packing algorithm which uses the cluster resource capacity or availability and multi-job estimated resource requirement information to provide better packing, such that more efficient resource utilization is achieved in the federated system. Specifically, the gang scheduling is adopted to co-schedule all the multi-jobs at a time, while the variable-sized multi-capacity bin-packing (VSMCBP) algorithm [22] places the jobs on nodes by colocating jobs tightly on each node. As multi-jobs arrive at the cluster $D_{\text {edge }_{\star}}$, the VSMCB algorithm scans the list of the

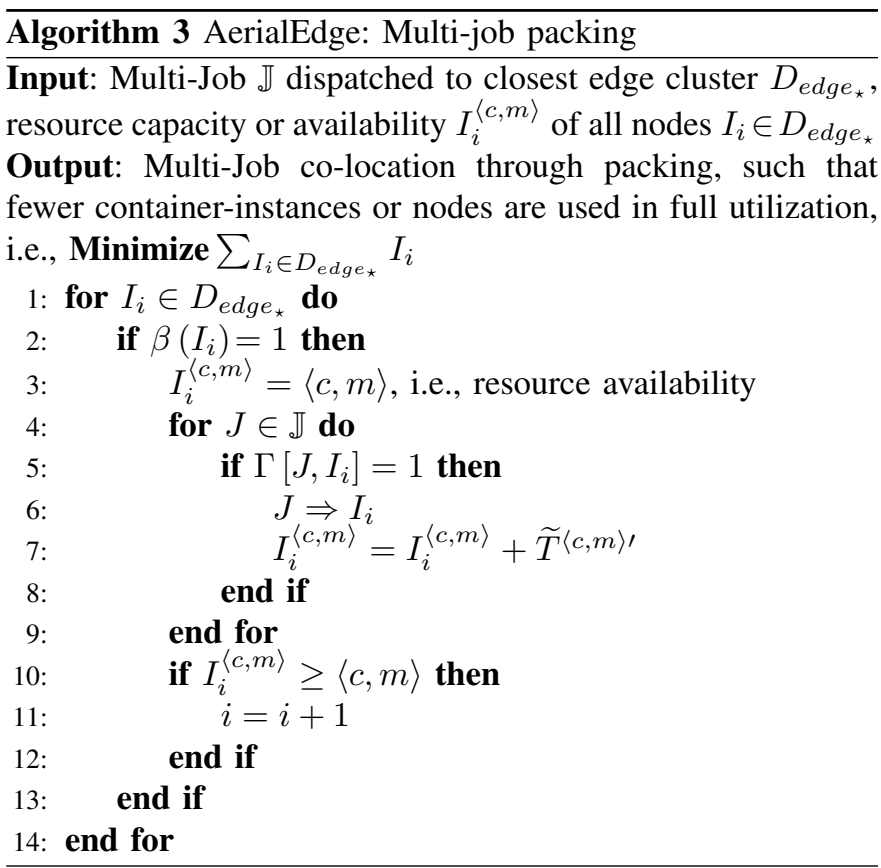

jobs, and maps these jobs to nodes. The key difference between the VSMCBP and other bin-packing algorithms, such as first fit bin packing (FFBP) [23], is the criteria used to select which jobs should be co-located to fully utilize any given node(s). The FFBP algorithm requires the next job to be packed on the current node, and if this cannot be done, a new node is used. The VSMCBP algorithm on the other hand scans the given list of jobs and maps jobs randomly to nodes in full utilization.

Multi-job $\mathbb{J}$ is a collection of several jobs $J \in \mathbb{J}$. These jobs are packed tightly on nodes, so that fewer nodes are used in full utilization and all the jobs are executed concurrently. Hence our packing strategy is to solve the problem:

$$
\begin{aligned}
\text { Minimize } & \sum_{I_{i} \in D_{\text {edge }}} I_{i}, \\
\text { subject to } & \mathbb{J} \Rightarrow D_{\text {edge }_{\star},} \\
& \sum_{J \in \mathbb{J}} \Gamma\left[J, I_{i}\right] \cdot \widetilde{T}^{\langle c, m\rangle \prime} \leq I_{i}^{\langle c, m\rangle}, \quad \forall_{c, m}, \\
& \Gamma\left[J, I_{i}\right]= \begin{cases}1, & \text { if } J \Rightarrow I_{i}, \\
0, & \text { otherwise }, \forall I_{i} \in D_{\text {edge }} .\end{cases}
\end{aligned}
$$

The constraint 25 indicates that the total estimated resource requirements of co-located jobs cannot exceed the node resource capacity or availability, while the condition $(26)$ means that if job $J$ is deployed on the node $I_{i}$, the indicator returns a value of 1 ; otherwise, 0 is returned. This is to ensure that each job is placed in exactly one node. The powerful Google OR-Tools [24], which provides an interface to several mixedinteger programming (MIP) solvers, i.e., coin-or branch and cut (CBC) [25], is employed to solve this VSMCBP problem for multi-job packing.

Algorithm 3 describes the packing strategy which packs tasks tightly on nodes, such that for any given tasks/jobs, fewer nodes are used for execution. It takes the estimated 
TABLE II: Federated-Edge Resource Capacities

\begin{tabular}{|c|l|c|c|c|}
\hline \hline Deployments & Attached Edge Devices & Total Weight & CPU Capacity & Mem Capacity \\
\hline Drone 1 & AWS Snowcone + Acer aiSage + Huawei AR502H & $3.5 \mathrm{~kg}$ & $12 \mathrm{Cores}$ & $8 \mathrm{GiB}$ \\
\hline Drone 2 & Lenovo ThinkSystem SE350 + Dell 3000s & $5.75 \mathrm{~kg}$ & $18 \mathrm{Cores}$ & $258 \mathrm{GiB}$ \\
\hline Drone 3 & HPE Edgeline EL300 + HIVECELL (x2) & $7.6 \mathrm{~kg}$ & $16 \mathrm{Cores}$ & $24 \mathrm{GiB}$ \\
\hline Drone 4 & INTELLIEDGE G700 + Azure Stack Edge mini + Dell 5000s & $9.6 \mathrm{~kg}$ & $26 \mathrm{Cores}$ & $72 \mathrm{GiB}$ \\
\hline Drone 5 & Azure Stack Edge mini $(x 2)+$ INTELLIEDGE G700 & $11.8 \mathrm{~kg}$ & $40 \mathrm{Cores}$ & $112 \mathrm{GiB}$ \\
\hline Drone 6 & Azure Stack Edge mini $(x 4)+$ HIVECELL $(x 2)$ & $15.4 \mathrm{~kg}$ & $76 \mathrm{Cores}$ & $208 \mathrm{GiB}$ \\
\hline
\end{tabular}

resource demand of multi-task/job and resource availability of container-instances or nodes as input, then scans through the multi-task/job to select jobs having congruent resources matching the active node in full utilization. This process is repeated until all jobs are scheduled on nodes.

\section{Performance Evaluation}

We evaluate our AerialEdge on real-time Alibaba cluster data traces, and compare its performance with three existing state-of-the-arts, and the Random approach.

\section{A. Setup}

Computing Resources: We use 6 distributed and federated aerial edge deployments (autonomous drones), as summarized in Table II The computing resources are made up of heterogeneous container-optimized nodes (container-instances). These drones have various resource capacities (up to $76 \mathrm{CPU}$ cores and $208 \mathrm{GiB}$ of memory) and weight (up to $15 \mathrm{~kg}$ ). We assume the selected drone has congruent flight time availability.

Applications: To evaluate our framework, we employ usecases of real-world CPU and memory intensive data-trace from Alibaba, which records the activities of both long running containers (for Alibabas e-commerce business) and batch jobs across an 8-day period. The data trace contains about 14,295,731 tasks (with about 12,207,703 dependencies) and 4,201,014 jobs, among which we randomly choose 46 jobs with total of 198 tasks (including dependencies) for our experiments.

\section{B. Heuristics and Baselines}

In our experiments, we assume that all tasks are of high priority. Our strategy utilizes the closest heuristic to minimize the overall response time. This is based on the orchestration of ready tasks to the closest drone deployment or cluster (i.e., with the smallest flight time to the needed location) having requisite flight time and available resources to execute the tasks. Closest is a widely adopted heuristic or principle in distributed systems, since mobile devices often need to communicate only with the closest or nearest edge-clouds. Most of the works on edge-clouds, e.g., [11], [21], adopt the closest principle as the task offloading policy.

In comparison of our AerialEdge with the state-of-the-art dependency-aware task orchestration and packing strategies, therefore, we fix the dispatching policy to that of AerialEdge, i.e., the closest heuristic. We compare our scheduling strategy with the following state-of-the-art benchmarks, and the Random approach.

1) Graphene [15] is a state-of-the-art approach for dependency-aware task orchestration problems. First, it co-schedules some tasks identified as troublesome tasks. Then the remaining tasks are divided into parent, child and sibling subsets, which are placed afterward to ensure compactness and to respect dependencies. It deploys about $40 \%$ of a given DAG at a time.

2) Tetris [12] is an existing state-of-the-art approach for task packing problems, although it does not consider the task dependencies. It deploys at least $50 \%$ of any given tasks at a time and primarily focuses on packing tasks on nodes mainly to achieve high resource utilization. For every task, it computes a packing score $\mathrm{pScore}_{t}$, as a dot product between the task resource requirements vector and the node's resource availability vector.

3) Spear [14] is a dependency-aware task scheduler, which applies Monte Carlo Tree Search (MCTS) with deep reinforcement learning. It utilizes the Critical Path (CP) to pick tasks along the $\mathrm{CP}$ in the DAG. Spear deploys about $15 \%$ of the tasks at a time.

4) Random approach deploys a task randomly to any available node, and assumes a node can only execute a task at a time.

\section{Deployment Results and Performance Comparison}

Our investigation focuses on CPU and memory usage/utilization, task deployment, scheduling and execution times. The multi-job execution information across the federated aerial edge deployments are listed in Table III The results obtained by AerialEdge, Graphene, Tetris, Spear and Random are compared.

1) Actual Resource Usage and Resource Utilization: We first introduce a performance metric by defining the actual resources usage of jobs $D_{e d g e_{i} A R U}^{\langle c, m\rangle}$ as the ratio of the resources used for execution $D_{\text {edge }_{i} U}^{\langle c, m)}$ over the edge's resource capacity or availability $D_{\text {edge }_{i}}^{\langle c, m\rangle}$ :

$$
D_{\text {edge }_{i} A R U}^{\langle c, m\rangle}=\frac{D_{\text {edge }_{i} U}^{\langle c, m\rangle}}{D_{\text {edge }_{i}}^{\langle c, m\rangle}} .
$$

Another metric is the resources utilization $\rho_{C}^{\langle c, m\rangle}$ given in 12, Similarly, $\rho_{C}^{\langle c, m\rangle}$ includes the CPU utilization $\rho_{C}^{\langle c\rangle}$ and the memory utilization $\rho_{C}^{\langle m\rangle}$, which are defined respectively by

$$
\begin{gathered}
\rho_{C}^{\langle c\rangle}=\frac{\sum_{J \in \mathbb{J}} \widetilde{T}^{\langle c\rangle}}{D_{\text {edge }_{i} U}^{\langle c\rangle}}, \\
\rho_{C}^{\langle m\rangle}=\frac{\sum_{J \in \mathbb{J}} \widetilde{T}^{\langle m\rangle \prime}}{D_{\text {edge }_{i} U}^{\langle m\rangle}},
\end{gathered}
$$


TABLE III: Multi-Task Execution in Federated Aerial Edge

\begin{tabular}{|c|c|c|c|c|c|}
\hline \hline$D_{\text {edge }_{i}}$ & $\mathbb{J}$ & $\mathbb{C}$ & $\gamma_{J}$ & $\widetilde{T}^{\langle c, m\rangle}$ & $\widetilde{E}_{\text {ex }}$ \\
\hline Drone 1 & 1 & 17 & 17 & $\langle 8.5,3.5\rangle$ & 384.9 \\
\hline Drone 2 & 3 & 24 & $(1,17]$ & $\langle 14.5,5.77\rangle$ & 456.23 \\
\hline Drone 3 & 5 & 16 & $(1,10]$ & $\langle 13.75,4.94\rangle$ & 506.75 \\
\hline Drone 4 & 8 & 26 & $(1,10]$ & $\langle 21.45,7.74\rangle$ & 609.9 \\
\hline Drone 5 & 13 & 38 & $(1,8]$ & $\langle 34,12.92\rangle$ & 387.83 \\
\hline Drone 6 & 16 & 77 & $(1.16]$ & $\langle 64.05,27.57\rangle$ & 1261.65 \\
\hline
\end{tabular}

where $\sum_{J \in \mathbb{J}} \widetilde{T}^{\langle c\rangle \prime}$ and $\sum_{J \in \mathbb{J}} \widetilde{T}^{\langle m\rangle \prime}$ are the total collective estimated CPU and memory demands, respectively.

Fig. 4 compare the resource usage of AerialEdge with the three baseline schemes, and the Random approach. It can be seen that AerialEdge and Tetris use the fewest resources in the clusters, better than Graphene, Spear and Random, while Spear and Random consume the highest resources in the clusters. The CPU and memory resource utilization comparisons are shown in Figs. 5 and 6, respectively. Again, AerialEdge and Tetris are superior than Graphene, Spear and Random, achieving the highest resource utilization, while Spear and Random achieve the lowest resource utilization. We now exam the individual clusters in detail. Fig. 7 shows the percentage of tasks deployed on each drone.

In Drone-1 edge-cluster, we deploy 1 job with a total of 17 tasks, where the job has a task dependency depth of 17.
AerialEdge first optimizes the deployment by co-locating as many jobs in a node as possible, to fully utilize the available resources in the node. Utilizing the gang scheduling strategy, AerialEdge co-schedules all the 17 tasks at a time. These tasks are tightly packed on nodes using the VSMCBP algorithm, which use $75 \%$ of the resources. Using the same configuration for the baseline schemes, Graphene and Tetris also use $75 \%$ of the resources while Spear use 92\%. The Random approach utilize all resources in the cluster. Importantly, AerialEdge gain faster scheduling and execution times compared to the three baseline schemes and the Random approach, mainly due to the following reasons: (i) AerialEdge utilizes the Gang Scheduling strategy, which co-schedules all the tasks at a time, and (ii) its Packing Strategy explores the available nodes to find the best nodes which have requisite available resources to execute all the tasks by packing them tightly on each node. Figs. 8 and 9 compare the actual scheduling and execution times respectively, of AerialEdge with the baseline schemes, and the Random approach. We observed that AerialEdge achieves faster scheduling and execution up to 42 times and 15 times respectively, compared with the state-of-the-art schemes. Compared with the Random approach, AerialEdge achieves faster scheduling and execution times of 393 times and 77

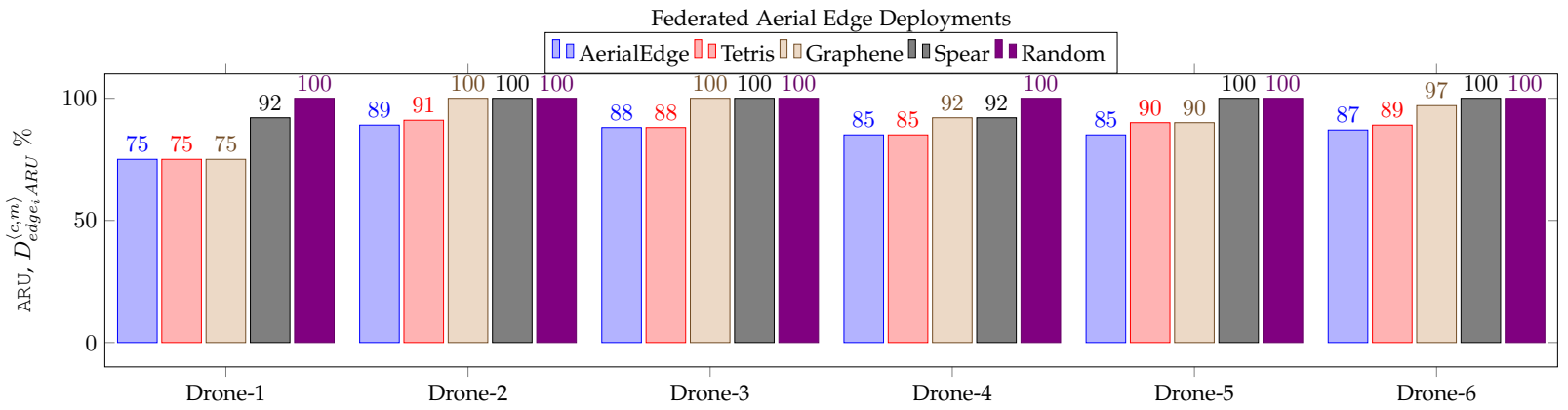

Fig. 4: Actual resource usage across the federated aerial edge clusters.

Federated 'Aerrial Edge Deployments

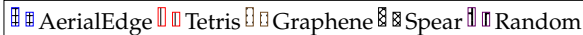

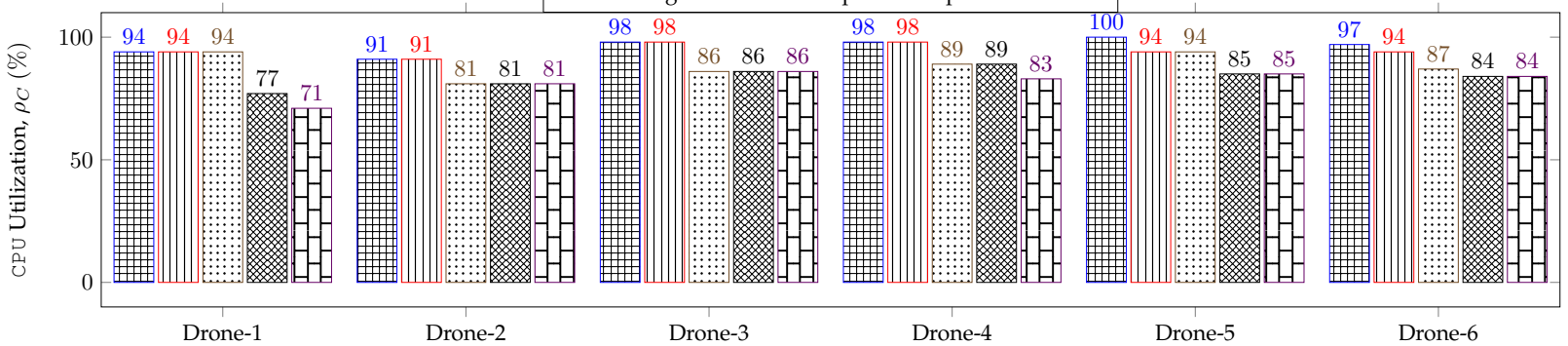

Fig. 5: CPU utilization across the federated aerial edge resources.

Federated Aerial Edge Deployments

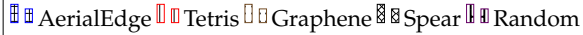

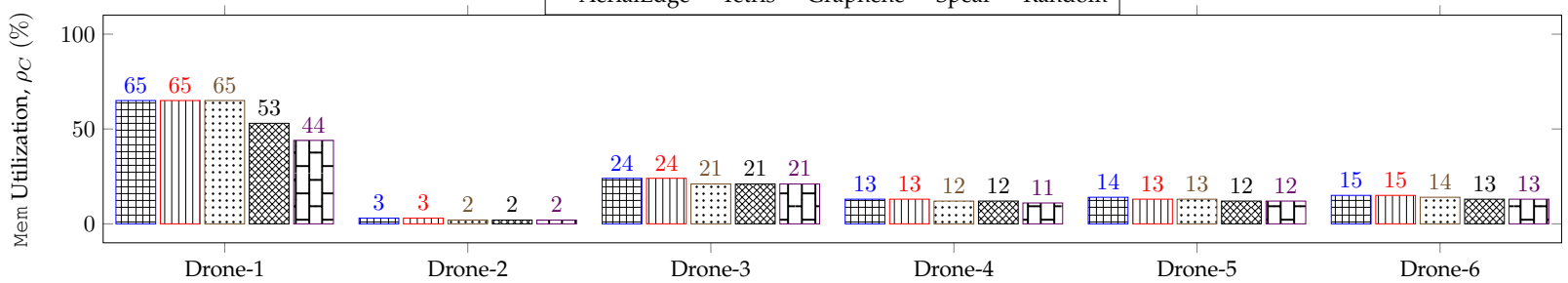

Fig. 6: Memory utilization across the federated aerial edge resources. 
times, respectively.

Drone-2 cluster is a memory intensive cluster. Here, 3 jobs with a total of 24 tasks are deployed, where each job has a task dependency in the range of $(1,17]$. We optimize the deployment to ensure that resources are fully utilized. AerialEdge consume $11 \%$ fewer resources than Graphene Spear and Random approach, and 2\% fewer resources than Tetris. AerialEdge and Tetris also gain 10\% higher CPU utilization over Graphene, Spear and Random, respectively, as well as $1 \%$ higher memory utilization than Graphene, Spear and Random, respectively. More significantly, AerialEdge is $1.1,2,2.7$ and 31 times faster in the scheduling time than Tetris, Graphene, Spear and Random, respectively, while it is 2, 4, 5 and 24 times faster in the execution time over these state-of-the-art schemes and Random approach, respectively. It is worth recapping that in the case of Random, the results of actual resource usage, resource utilization, scheduling and execution times are for $38 \%$ of the tasks that it is able to deploy on Drone-2.

Drone-3 has a total load of $7.6 \mathrm{~kg}$, and it is made up of $1 \mathrm{HPE}$ Edgeline EL300 and 2 HIVECELL edge devices, with total resource capacity of 16 Cores and $24 \mathrm{GiB}$. In this cluster, we deploy 5 jobs, with total 16 tasks, where each job has a task dependency range $(1,10]$. AerialEdge and Tetris reduce resource usage by $12 \%$ compared with Graphene, Spear and Random. AerialEdge and Tetris achieve $12 \%$ and 3\% higher CPU and memory utilization, respectively, compared to Graphene, Spear and Random. In terms of both scheduling and execution times, AerialEdge is about 4.5 and 2.2 times faster than Tetris. It is 6.7 times and 3.4 times faster than Graphene as well as 13 times and 5.5 times faster than Spear, in the scheduling and execution times, respectively. Not surprisingly, Random has the worst scheduling and execution time performance.

Drone-4 cluster is a high capacity cluster. Here, 8 jobs with total of 26 tasks are deployed, where each job has a dependency depth range $(1,10]$. It can be seen that AerialEdge and Tetris consume 7\% fewer resources than Graphene and Spear. AerialEdge and Tetris also achieve 9\% higher CPU utilization as well as $1 \%$ higher memory utilization, over Graphene and Spear, respectively. Random can only deploy $50 \%$ of the tasks. By contrast, AerialEdge, Tetris, Graphene and Spear all deploy $100 \%$ of the tasks. In terms of scheduling time, AerialEdge is approximately 2 times, 3.2 times , 5.3 times, and 41.3 times faster than Tetris, Graphene, Spear and Random, respectively. In terms of execution time, AerialEdge

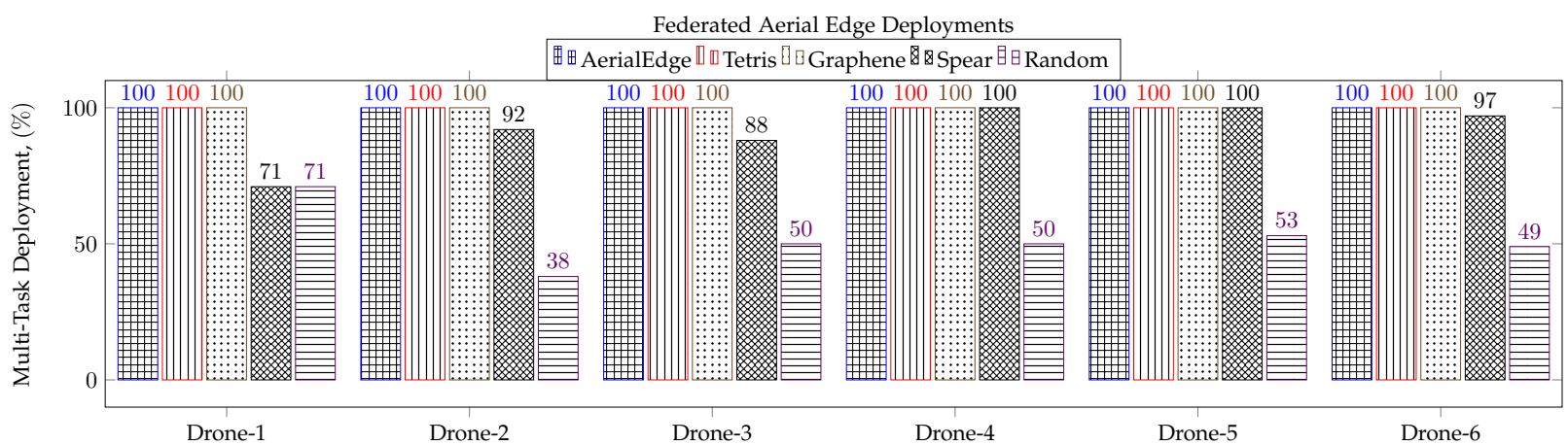

Fig. 7: Multi-task deployment across the federated aerial edge resources.

Federated Aerial Edge Deployments

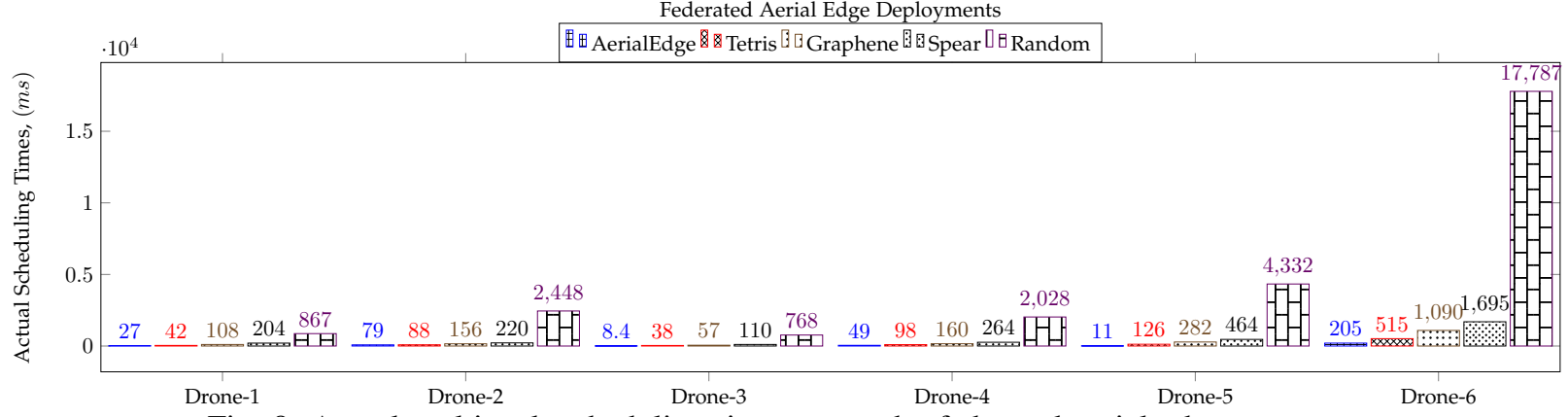

Fig. 8: Actual multi-task scheduling time across the federated aerial edge resources.

Federated Aerial Edge Deployments

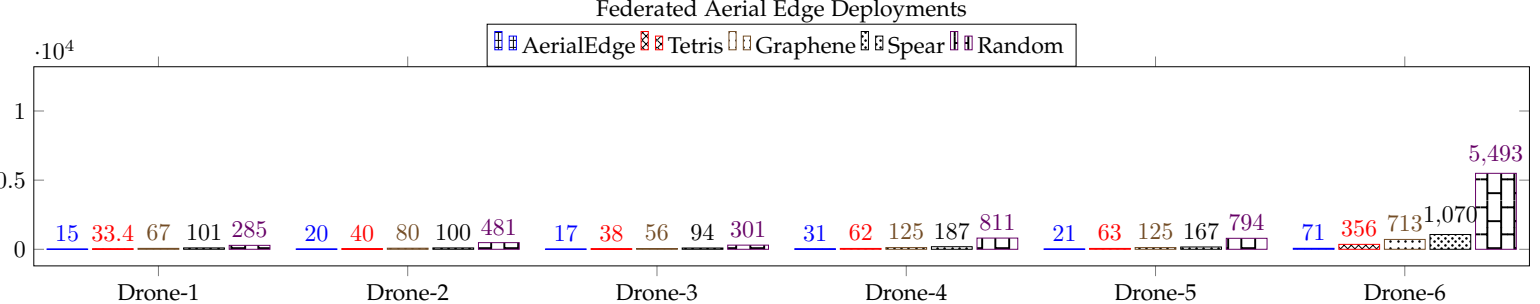

Fig. 9: Actual multi-task execution time across the federated aerial edge resources. 
is about 2 times, 4 times, 6 times and 26 times faster than Tetris, Graphene, Spear and Random, respectively. Again Random has the worst scheduling and execution time performance.

Drone -5 and Drone-6 are the largest clusters in terms of resource and load capacity. We deploy 13 and 36 jobs in these two clusters, respectively. The total number of tasks deployed in Drone -5 cluster is 38 , while total of 77 tasks are deployed in Drone-6 cluster. The task dependency depth of each job is in the range of $(1,16]$. In these two clusters, AerialEdge use up to $13 \%$ less resources compared with the baselines and the Random approach. Specifically, Spear and Random used up all available resources, with some nodes running underutilization. AerialEdge and Tetris achieve the highest resource utilization and deploy $100 \%$ of the tasks in both clusters. Random approach could only deploy $53 \%$ and $49 \%$ of all tasks in Drone-5 and Drone-6, respectively. We observed that AerialEdge achieves faster scheduling and execution of up to 42 times and 15 times than the baseline schemes in Drone-5 and Drone-6, and faster scheduling and execution of up to 393 times and 77 times than the Random approach in both clusters.

2) Task Scheduling and Execution Times: The task scheduling time, which is the time it takes to place multi-jobs/tasks on the nodes in a cluster, is an important performance metric to assess the federated edge clusters. Another even more important performance metric is the aggregate job actual execution time $E_{e x} /$ defined in $(10)$. Figs. 8 and 9 compare the scheduling times and execution times, respectively, attained by the four schemes. It can be seen that the scheduling times are typically very small, and the execution times by contrast are significantly larger. Across the federated edge clusters, AerialEdge consistently achieves the fastest scheduling and execution times, compared to the three benchmark strategies, and the Random approach.

\section{Conclusions}

This paper has presented a dependency-aware multi-task orchestration in a federated aerial edge computing-enabled learning system, called AerialEdge, to improve resource efficiency and enhance performance. Our approach involves multi-task resource requirements and execution time estimations, federated aerial edge clusters update state service, gang scheduling and co-location on container-optimized nodes. We have compared our approach with the state-of-the-art dependency-aware task orchestration and task packing baseline strategies. AerialEdge achieves both the highest cluster resource utilization and the minimum execution time for multitasks/jobs compared to the baseline strategies. We observe that AerialEdge consumes up to $25 \%$ fewer resource and achieves up to $23 \%$ high cluster utilization, while leading to up to 393 times faster scheduling time and up to 77 times faster execution time.

\section{ACKNOWLEDGMENT}

The financial support of the National Natural Science Foundation of China under grant 61571401 and the Innovative
Talent of Colleges and the University of Henan Province under grant 18HASTIT021 are gratefully acknowledged.

\section{REFERENCES}

[1] AWS Snowcone, Accessed on July 10, 2021. [Online]. Available: https://aws.amazon.com/snowcone/

[2] HIVECELL, Accessed on July 10, 2021. [Online]. Available: https://hivecell.com/hardware/

[3] Drone-in-a-box, Accessed on July 10, 2021. [Online]. Available: https://en.wikipedia.org/wiki/Drone_in_a_Box

[4] F. Giuseppe, G. Christian and S. Giovanni "Fog in the clouds: UAVs to provide edge computing to IoT devices," ACM Trans. Internet Technology, vol. 20, no. 3, pp. 26:1-26:26, 2020

[5] J. Ren, et al., "Federated Learning-Based Computation Offloading Optimization in Edge Computing-Supported Internet of Things," IEEE Access, vol. 7, pp. 69194-69201, 2019.

[6] R. Yu and P. Li, "Toward Resource-Efficient Federated Learning in Mobile Edge Computing," IEEE Network, vol. 35, no. 1, pp. 148-155, January/February 2021.

[7] B. McMahan, et al., "Communication-Efficient Learning of Deep Networks from Decentralized Data," Proc. AISTATS 2017 (Fort Lauderdale, FL, USA), Apr. 20-Apr. 22, pp. 1273-1282.

[8] U. Awada and J. Zhang, "Edge Federation: A Dependency-Aware Multi-Task Dispatching and Co-location in Federated Edge ContainerInstances," in Proc. EDGE 2020 (Beijing, China), Oct. 19-Oct. 23, pp. 91-98.

[9] R. Urgaonkar, et al., "Dynamic service migration and workload scheduling in edge-clouds," Performance Evaluation, vol. 91, pp. 205-228, 2015.

[10] L. Tong, Y. Li, and W. Gao, "A hierarchical edge cloud architecture for mobile computing," in Proc. INFOCOM 2016 (San Francisco, CA, USA), Apr. 10-14, 2016, pp. 1-9.

[11] U. Awada and A. Barker, "Improving resource efficiency of containerinstance clusters on clouds," in Proc. CCGRID 2017 (Madrid, Spain), May 14-17, 2017, pp. 929-934.

[12] R. Grandl, et al., "Multi-resource packing for cluster schedulers," in Proc. SIGCOMM 2014 (Chicago, IL, USA), Aug. 17-22, 2014, pp. 455466.

[13] Alibaba Cluster Trace, Accessed on July 10, 2021. [Online]. Available https://github.com/alibaba/clusterdata/blob/master/ cluster-trace-v2018/trace-2018.md

[14] Z. Hu, J. Tu, and B. Li, "Spear: Optimized dependency-aware task scheduling with deep reinforcement learning," in Proc. ICDCS 2019 (Dallas, TX, USA), Jul. 7-10, 2019, pp. 2037-2046.

[15] R. Grandl, et al., "GRAPHENE: Packing and dependency-aware scheduling for data-parallel clusters," in Proc. OSDI 2016 (Savannah, GA, USA), Nov. 2-4, 2016, pp. 81-97.

[16] Z. Han, et al., "Scheduling placement-sensitive BSP jobs with inaccurate execution time estimation," in Proc. INFOCOM 2020 (Toronto, ON, Canada), Jul. 6-9, 2020, pp. 1053-1062.

[17] Keras, Accessed on July 10, 2021. [Online]. Available: https://keras.io/

[18] TensorFlow, Accessed on July 10, 2021. [Online]. Available: https://www.tensorflow.org/

[19] M. Mukherjee, et al., "Distributed deep learning-based task offloading for UAV-enabled mobile edge computing," in Proc. INFOCOM WKSHPS 2020 (Toronto, ON, Canada), Jul. 6-9, 2020, pp. 1208-1212.

[20] Y. Liu, S. Xie and Y. Zhang, "Cooperative offloading and resource management for UAV-enabled mobile edge computing in power IoT system," IEEE Trans. Vehicular Technology, vol. 69, no. 10, pp. 12229$12239,2020$.

[21] Z. Han, et al., "OnDisc: Online latency-sensitive job dispatching and scheduling in heterogeneous edge-clouds," IEEE/ACM Trans. Networking, vol. 27, no. 6, pp. 2472-2485, 2019.

[22] P. Lai, et al., "Optimal edge user allocation in edge computing with variable sized vector bin packing," in Proc. ICSOC 2018 (Hangzhou, China), Nov. 12-15, 2018, pp. 230-245.

[23] S. Rampersaud and D. Grosu, "Sharing-Aware Online Virtual Machine Packing in Heterogeneous Resource Clouds," in IEEE Transactions on Parallel and Distributed Systems, vol. 28, no. 7, pp. 2046-2059, 2017.

[24] Google OR-Tools, Accessed on July 10, 2021. [Online]. Available: https://developers.google.com/optimization

[25] Coin-or branch and cut, Accessed on July 10, 2021. [Online]. Available: https://projects.coin-or.org/Cbc 